\title{
Structural basis of peptide-carbohydrate mimicry in an antibody-combining site
}

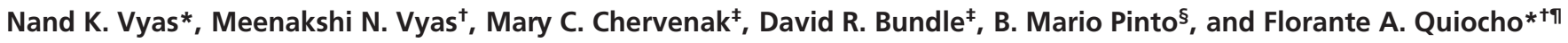 \\ *Department of Biochemistry and Molecular Biology and ${ }^{\dagger}$ Howard Hughes Medical Institute, Baylor College of Medicine, Houston, TX 77030; ${ }^{\star}$ Department \\ of Chemistry, University of Alberta, Edmonton, AB, Canada T6G 2G2; and §Departments of Chemistry and of Molecular Biology and Biochemistry, \\ Simon Fraser University, Burnaby, BC, Canada V5A 1 S6
}

Edited by David S. Eisenberg, University of California, Los Angeles, CA, and approved October 1, 2003 (received for review March 5, 2003)

The structure of a complex between the Fab fragment of the antibody (SYA/J6) specific for the cell surface O-antigen polysaccharide of the pathogen Shigella flexneri $Y$ and an octapeptide (Met-Asp-Trp-Asn-Met-His-Ala-Ala), a functional mimic of the $\mathrm{O}$-antigen, has been determined at 1.8-Å resolution. Comparison of the structure with that of the complex with the pentasaccharide antigen $[\rightarrow 2)$ - $\alpha$-L-Rha- $(1 \rightarrow 2)$ - $\alpha$-L-Rha-( $1 \rightarrow 3)$ - $\alpha$-L-Rha-(1 $\rightarrow 3)-\beta$-D-GlCNAc- $(1 \rightarrow 2)-\alpha$-L-Rha- $(1 \rightarrow]$ reveals the molecular recognition process by which a peptide mimics a carbohydrate in binding to an antibody. The binding modes of the two ligands differ considerably. Octapeptide binding complements the shape of the combining site groove much better than pentasaccharide binding. Moreover, the peptide makes a much greater number of contacts (126), which are mostly van der Waals interactions, with the Fab than the saccharide (74). An unusual feature is also the involvement of 12 water molecules in mediating hydrogen bonds between residues within the peptide or of the peptide and Fab. Despite better shape complementarity and greater number of contacts, the octapeptide binds with an affinity $\left(K_{\mathrm{A}}=2.5 \times 10^{5} \mathrm{M}^{-1}\right.$, measured by calorimetry) only $\approx 2$-fold tighter than the pentasaccharide. The structural results are relevant to the design of peptide mimetics with improved affinity for use as vaccines.

$\mathbf{T}^{\mathrm{h}}$ he cell-surface polysaccharides of pathogenic bacteria and viruses are important virulence factors and antigens and are therefore excellent targets for vaccine development (1). Furthermore, oligosaccharide antigens in the form of synthetic vaccines are being intensely investigated as an auxiliary treatment of cancer (2). Most polysaccharides, although immunogenic in adults, do not induce any immune response in children $<2$ years of age and induce a $\mathrm{T}$-independent response that lacks immunological memory (1). The approach may also fail in cases where carbohydrate primary structures are similar to those of the human host (1).

One successful strategy to overcome these difficulties has been the conjugation of carbohydrates to proteins or $\mathrm{T}$ cell peptides. These glycoconjugate vaccines elicit $\mathrm{T}$-dependent responses and may be used directly as immunogens or as priming agents for boosting with polysaccharide $(3,4)$. Carbohydrate-mimetic peptides are seen as another possible solution (reviewed in ref. 5). Although they possess unquestionable advantages as immunogens, the success of this strategy crucially depends on the ability of peptides to mimic oligosaccharide epitopes.

Peptides that crossreact with carbohydrates may be identified by screening of phage-displayed peptide libraries with anticarbohydrate antibodies (6). Their binding affinities are often similar to or greater than those of the native ligands, and consensus sequences for groups of crossreactive peptides have been identified $(5,6)$. These peptides are lead candidates for vaccine development $(7,8)$. When used as immunogens, they may elicit carbohydrate-binding antibody responses $(7,8)$. The design of an effective vaccine that elicits an antipolysaccharide response, based on a peptide mimetic that is complementary to only one monoclonal $\mathrm{Ab}$, has precedent in the case of a vaccine against Group B Streptococcus polysaccharide, based on carbohydrate-mimetic peptides (9).

The question of whether carbohydrate-mimetic peptides that elicit carbohydrate-binding antibodies are structural mimics of the carbohydrates, or merely functional mimics that may interact with the antibody-combining site in a different manner, is fundamental to our understanding of mimicry at the molecular level. Whether either structural or functional mimicry is a prerequisite for mimetic peptides to elicit carbohydrate-binding antibodies, and thus for vaccine development $(5,7,8)$, is a further question that remains to be probed. As a first step in this program, we present structural evidence that shows that the mechanism of peptide binding differs from that of carbohydrate in the binding to an antibody.

We chose to study the anti-Shigella monoclonal antibody SYA/J6 (10) Fab complexed with a peptide mimetic, because the corresponding structures and thermodynamics of complexes with carbohydrate $\mathrm{O}$-antigens were recently available for comparison (11). SYA/J6 is a murine $\mathrm{IgG}_{3}(\kappa)$ antibody specific for the polysaccharide O-antigen of the Shigella flexneri Y lipolysaccharide. The polysaccharide is a linear heteropolymer $[\rightarrow 2)-\alpha$ L-Rha- $(1 \rightarrow 2)-\alpha$-L-Rha- $(1 \rightarrow 3)-\alpha$-L-Rha- $(1 \rightarrow 3)-\beta$-D-GlcNAc$(1 \rightarrow 2)-\alpha$-L-Rha- $(1 \rightarrow]$ (or Rha A-Rha B-Rha C-GlcNAc D). We report here the $3 \mathrm{D} x$-ray structure and thermodynamics of the antibody Fab fragment with the bound octapeptide, Met P1-Asp P2-Trp P3-Asn P4-Met P5-His P6-Ala P7-Ala P8 (P stands for peptide), discovered by screening of phage-displayed libraries (12). Comparison of the structure with that previously determined of the complex with a pentasaccharide (Rha A-Rha B-Rha C-GlcNAc D-Rha A') (11) identifies for the first time the atomic details of the recognition process by which a peptide mimics a carbohydrate in the binding of an antibody.

\section{Materials and Methods}

Material. The octapeptide MDWNMHAA $\left(M_{\mathrm{r}}=975.1\right)$ was synthesized as its carboxamide (Alberta Peptide Institute, Edmonton, Canada). It represents a portion of the phagedisplayed peptide sequence MDWNMHAAEGDD isolated by the monoclonal anti-carbohydrate antibody SYA/J6 (12). The Fab of SYA/J6 (IgG3, $\kappa$, murine) used in this study was prepared as described (13).

X-Ray Structure Determination. Because the Fab-octapeptide complex failed to be cocrystallized, it was formed by soaking ligand-free crystals of the Fab (13) in solution containing the octapeptide. The ligand-free Fab crystals, which were harvested in $65 \%$ 2-methyl-2,4-pentanediol/0.02\% sodium azide/25 mM

This paper was submitted directly (Track II) to the PNAS office.

Abbreviations: $C D R$, complementarity-determining region; $\mathrm{VH}$, variable heavy chain; $\mathrm{VL}$, variable light chain

Data deposition: The coordinates have been deposited in the Protein Data Bank, www. rcsb.org (PDB ID code 1PZ5).

"To whom correspondence should be addressed. E-mail: faq@bcm.tmc.edu.

C 2003 by The National Academy of Sciences of the USA 
Table 1. Statistics for $x$-ray data collection and refinement of the crystal structure of the SYA/J6 Fab-octapeptide complex

\begin{tabular}{|c|c|}
\hline Item & Fab-peptide \\
\hline Space group & $P 4_{3} 2_{1} 2$ \\
\hline Unit cell parameters, Å & $\begin{array}{c}a=b=69.3 \\
c=198.7\end{array}$ \\
\hline Mosaicity & 0.65 \\
\hline Resolution range, $\AA$ & $20.0-1.8$ \\
\hline Total measured reflections, $F>0.0 \sigma_{F}$ & $169,601(9,660)^{*}$ \\
\hline Unique reflections, $F>0.0 \sigma_{F}$ & $42,148(2,644)^{*}$ \\
\hline Redundancy & $4.02(3.65)^{*}$ \\
\hline$I / \sigma_{1}>20, \%$ & $50(2) *$ \\
\hline Completeness, \% & $91.7(58.9) *$ \\
\hline$R_{\text {merge, }} \%$ & $4.2(12.0)^{*}$ \\
\hline$R_{\text {cryst, }}, F>0.0 \sigma_{F}$ & $0.219(0.233)^{\dagger}$ \\
\hline$R_{\text {free, }}, 10 \%$ data, $F>0.0 \sigma_{F}$ & $0.254(0.268)^{\dagger}$ \\
\hline \multicolumn{2}{|l|}{ No. of residues or solvents } \\
\hline rms deviation & $435 / 8 / 519$ \\
\hline Bond distance, $\AA$ & 0.006 \\
\hline Bond angles, ${ }^{\circ}$ & 1.5 \\
\hline \multicolumn{2}{|l|}{ Ramachandran analysis, \% } \\
\hline Most favored regions & 90.0 \\
\hline Additionally allowed regions & 9.6 \\
\hline Generally allowed regions & 0.4 \\
\hline
\end{tabular}

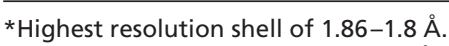

†Highest resolution shell of 1.91-1.8 $\AA$.

sodium phosphate ( $\mathrm{pH} 7.2$ ), were soaked in the same solution containing $1 \mathrm{mM}$ peptide. The crystals were frozen by being placed in nitrogen gas stream cooled at $-170^{\circ} \mathrm{C}$. Initial proof of the binding of the octapeptide was provided by its well resolved density in a $3-\AA$ resolution difference Fourier $\left(F_{\mathrm{o}}-F_{\mathrm{c}}\right)$ map calculated with the sugar-free Fab structure phases (11). The intensity data set was collected by using DIP2030 (MacScience, Yokohama, Japan) area detector mounted on a RU200 rotating anode (Rigaku, Tokyo, Japan). Diffraction data to 1.8 - $\AA$ resolution were then collected at $-170^{\circ} \mathrm{C}$ at the Howard Hughes Medical Institute Brookhaven National Laboratory beamline X4A. The wavelength was $0.948 \AA$, the crystal to detector (RAXIS-IV) distance was $300 \mathrm{~mm}$, and the data were collected at $1^{\circ}$ oscillations. The data were reduced and scaled by using DENZO/SCALEPACK programs (14). Diffraction statistics are shown in Table 1.

Because the crystal of the Fab-peptide complex is isomorphous to that of the native Fab (11), its crystal structure was determined by direct phasing with the structure of the native Fab. The CNS suite of programs (15) was used in the structure determination and refinement (Table 1). The structure of the Fab-peptide complex was initially refined by the rigid-body method by using the unliganded coordinates of native Fab (11) and data to $3 \AA$. The density of the octapeptide in the antigenbinding site is clearly evident in the $\left(F_{\mathrm{o}}-F_{\mathrm{c}}\right)$ and $\left(2 F_{\mathrm{o}}-F_{\mathrm{c}}\right)$ Fourier maps, allowing easy fitting of its known sequence. After inclusion of the peptide in the structure-refinement process, the resolution was extended incrementally up to $2 \AA$ by using the method of slow annealing (alternate cycles of positional and B factor parameters refinement). At this stage water molecules were identified and incorporated in the final round of refinements to $1.8 \AA$; the final statistics shown in the Table 1 . The geometry of the structure was validated by PROCHECK (16). The segment of residues H128-H135 shows no interpretable density, which is typically observed in Fab structures, including the previous three SYA/J6 Fab structures (11).
Illustrations. The following programs were used for making the figures: MIDAS (17) for Figs. $1 A$ and $2 B$, GRASP (18) for Fig. $1 B$ and $C$, and MOLSCRIPT (19) for Figs. $2 A$ and 4.

Titration Microcalorimetry. Calorimetric measurements were made by using the Microcal MCS titration microcalorimeter (Microcal, Northampton, MA) and following a similar procedure and data analysis used in the binding of oligosaccharide antigen to SYA/J6 antibody (11). The concentration of the antibody was measured by UV and that of the peptide was calculated based on quantitative amino acid analysis composition. The antibody $(20.6 \mu \mathrm{M})$ was placed in the cell maintained at $25^{\circ} \mathrm{C}$ and titrated with the octapeptide to a final concentration of at least $10 \times K_{\mathrm{A}}$. Titrations of the antibody were carried out at $\mathrm{pH} 8.0$ in $50 \mathrm{mM}$ Tris buffer augmented with $150 \mathrm{mM}$ $\mathrm{NaCl} / 0.02 \% \mathrm{NaN}_{3}$. Typically, thirty $8-\mu$ l injections of peptide $(0.245 \mathrm{mM})$ maintained at $25^{\circ} \mathrm{C}, 20 \mathrm{~s}$ in duration, were made, with 4-min intervals between injections. Each injection generated a measurable heat from the association of protein and ligand. The data were integrated to provide a titration curve; and, by using a nonlinear least-squares fit, the binding constant $K_{\mathrm{A}}$, the heat of binding $(\Delta H)$, and the stoichiometry of binding (one bound ligand) were extracted from the curve. Nonnegligible heats of dilution were subtracted before data processing.

\section{Results and Discussion}

Overall Fab-Octapeptide Complex Structure. The crystal structure of the complex was refined to 1.8 - $\AA$ resolution, an $R_{\text {cryst }}$ of 0.219 , an $R_{\text {free }}$ of 0.254 , and good geometry (Table 1$)$. The combining site in the Fab is a deep groove that runs parallel to the interface of the variable light chain (VL) and variable heavy chain $(\mathrm{VH})$ domains (Fig. 1). The groove is $\approx 25 \AA$ long by $12 \AA$ wide by 10 $\AA$ deep, sloping to a cavity or pocket in the bottom. The residues of complementarity-determining region (CDR) H3 and L3 line mostly the floor of the groove, whereas CDR L1/L2 and CDR $\mathrm{H} 1 / \mathrm{H} 2$ form the two sides. As originally revealed by the first structures of the SYA/J6 Fab (11), the combining site exhibits a predominantly negative electrostatic surface potential. Despite this feature, the octapeptide selected in the phage display contains only one potential basic residue (His P6).

The octapeptide, with very well resolved electron density (Fig. $2 A$ ), fits into the groove with the first four residues (Met P1-Asp P2-Trp P3-Asn P4) in a semiextended conformation and the last four residues (Met P5-His P6-Ala P7-Ala P8) in a 3.6 13 -turn conformation as in an $\alpha$-helix (Figs. $1 A$ and 2). It is involved in numerous interactions, including the following types of hydrogen bonds (Figs. $2 B$ and $3 A$ ): (i) direct intramolecular hydrogen bonds between residues of the octapeptide, (ii) direct intermolecular hydrogen bonds between the Fab and the peptide, (iii) water-mediated hydrogen bonds within the octapepeptide, and (iv) water-mediated hydrogen bonds between the Fab and the peptide. Moreover, many van der Waals contacts mediate the Fab-peptide complex (Fig. 3C).

Five direct intramolecular hydrogen bonds are formed between mostly main-chain polar groups of five residues within the octapeptide, which stabilize the $\alpha$-helical turn (Fig. 2B). Six direct intermolecular hydrogen bonds are made between mainly side chains of three peptide and five Fab residues (Fig. $3 A$ ). At least 14 ordered water molecules are associated with octapeptide binding, of which 12 are involved in a web of hydrogen bonds within the octapeptide (Fig. $2 B$ ) and between the peptide and the Fab (Fig. $3 A$ ).

Octapeptide Binding. The binding of the peptide can be divided in relation to portions of the groove contacted by three segments: Met P1-Asp P2, Trp P3-Asn P4-Met P5 and His P6-Ala P7-Ala $\mathrm{P} 8$. The $\mathrm{N}$ terminus of the peptide is located at one end of the antigen-binding groove (Fig. $1 A$ and $B$ ), near where the reducing 

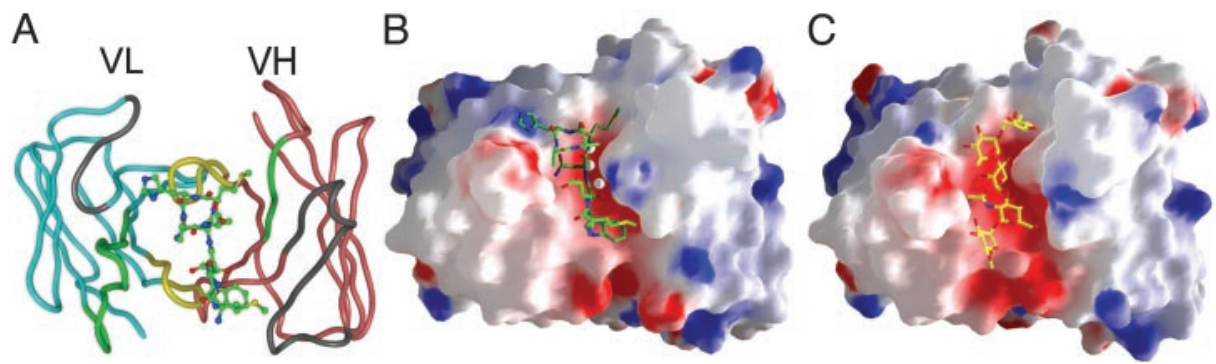

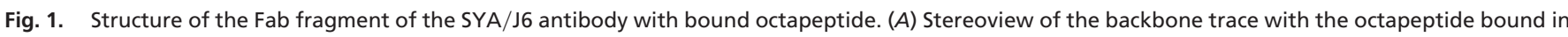

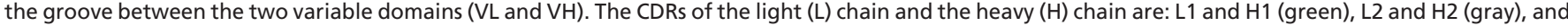

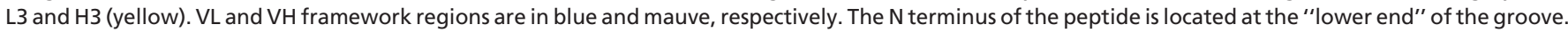

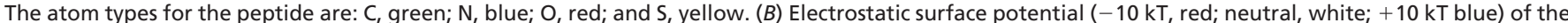

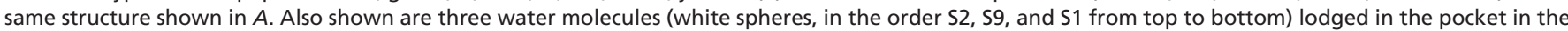

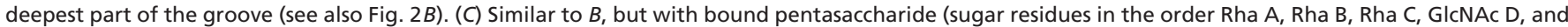
Rha $A^{\prime}$ from top to bottom) (data from ref. 11). The atom types for the sugar are: $C$, yellow; N, blue; and $O$, red. Rha $C$ mainly occupies the groove pocket.

end (Rha $\mathrm{A}^{\prime}$ ) of the pentasaccharide binds (Fig. 1C) (11). Here, the side chains of Met P1-Asp P2 are partly exposed to solvent and interact with the Fab through only two hydrogen bonds and very few van der Waals interactions (Fig. $3 A$ and $C$ ). However, both residues are engaged in a much greater number of watermediated hydrogen bonds, five intramolecular and six intermolecular types (Figs. $2 B$ and $3 A$ ).

The next three residues (Trp P3-Asn P4-Met P5) lie in the middle part of the groove (Fig. $1 A$ and $B$ ), where the saccharides Rha $\mathrm{C}$ and GlcNAc D are lodged (Fig. $1 C$ ). Like the two sugar residues (11), the three peptide residues are the most buried segment of the octapeptide and the most involved in both nonpolar and polar interactions with the combining site (Figs. 3 $A$ and $C$ and $4 A$ ). This finding is consistent with the previous finding that Trp P3 and Asn P4 are indispensable (20). The indole ring of Trp P3 occupies a shallow hydrophobic cavity on the surface of the groove (Fig. $1 B$ ) formed mainly by residues from CDR L3 (Val L94 and Pro L95), CDR H2 (His H58), and a framework residue (Trp H47) (Fig. 4A). With the side chain of Met P1 lying across one face of the indole ring, the indole is fully enclosed. The substantial hydrophobic interactions involving Trp P3 and Met P1 are the first of two other substantial nonpolar interactions (Fig. $4 B$ and $C$ ). By engaging in a total of eight hydrogen bonds (four intramolecular; Fig. $2 B$ ), two intermolecular and two water-mediated hydrogen bonds (Fig. 3A), Asn P4 is the peptide residue most heavily involved in maintaining the
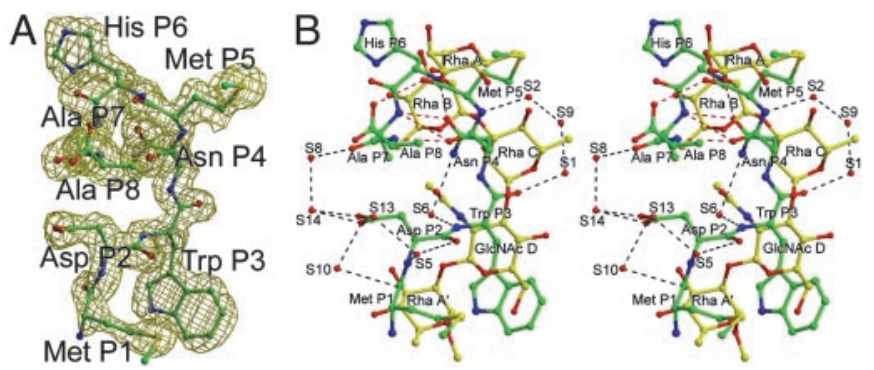

Fig. 2. The bound octapeptide. (A) 1.8 - $\AA$ resolution sigma-weighted $\left(2 F_{0}-\right.$ $F_{\mathrm{c}}$ ) map of the octapeptide contoured at $1 \sigma$ level. $(B)$ Stereoview of the superimposed bound pentasaccharide (11) and octapeptide. This figure is related to those in Fig. 1 by a $90^{\circ}$ rotation about the groove (vertical) axis. In this lateral view, the opening of the groove is to the left and the bottom to the right. Relative to Fig. $1 B$ and $C$, the deep pocket of the combining site groove is filled by $\mathrm{S2}, \mathrm{S}$, and $\mathrm{S} 1$ and mainly by Rha C, respectively. Also shown are nine solvents $(\mathrm{S})$ that bridge five hydrogen-bonding interactions between residues within the octapeptide. Not shown are water molecules S4 and S7, which bind solely to the peptide. peptide conformation and in interacting with the Fab. The hydrogen bonds of the side-chain carbonyl oxygen would favor formation of the $\alpha$-helical turn observed in the following four residues; "helix initiation" in this manner is an important function of Asn in proteins (21). The nonpolar side chain of Met P5 lies in a shallow nonpolar cavity and almost across one side of the indole ring of Trp H33, which in turn makes a cation- $\pi$ interaction (22) with Arg H52 (Figs. $1 B$ and $4 B$ ).

The $\alpha$-helical turn enables the last three peptide residues (His P6-Ala P7-Ala P8) to form polar and nonpolar interactions in the other end of the groove (Figs. $1 B, 3 A$, and $4 C$ ). Like the first two residues, the last two are partially exposed to the solvent. All three residues are engaged in only two direct and two watermediated hydrogen bonds with the Fab. The hydrogen bond and other contacts between His P6 side chain and Lys L50 NZ, the first residue of the short loop CDR L2, are unusual in that this loop makes no contact with the saccharide in this or other complexes of antibody with carbohydrates $(23,24)$ or haptens $(25,26)$. The Ala P7 methyl side chain lies close to, but not completely inside, a small hydrophobic cavity (Tyr L32 and His L27D) (Fig. 4C).

Comparison Between Octapeptide and Pentasaccharide Binding. The accessible surface area of the bound peptide $\left(1,087 \AA^{2}\right)$ is only $6 \%$ greater than that of the bound pentasaccharide $\left(1,029 \AA^{2}\right)$ when measured in the absence of protein and solvent atoms. Nevertheless, the bound peptide is significantly more exposed to the solvent (accessible surface of $451 \AA^{2}$ ) than the bound pentasaccharide (accessible surface area of $335 \AA^{2}$ ).

Superposition of the two complex structures yielded additional observations that highlight the major differences in the binding between the octapeptide and pentasaccharide. First, the octapeptide makes significantly more direct contacts with the Fab [total of 126 of $\leq 4 \AA$, including six $\mathrm{H}$ bonds (Fig. $3 A$ and $C)$ ] than the pentasaccharide [74 contacts, including eight $\mathrm{H}$ bonds (Fig. $3 B$ and $C)$ ] (11). Most of the contacts $(\approx 57 \%)$ with both ligands are made with the light chain. Most of the contacts between the peptide and the L chain are with the CDR L3 loop (Fig. 1A), which forms a shallow hydrophobic cavity that harbors mainly Trp P3 near the N-terminal end of the octapeptide (Fig. $1 B$ ). This cavity is not harnessed in pentasaccharide binding (Fig. $1 C)$. Further indications that a large majority of the contacts differ between the two ligands are as follows. Of only 37 contacts with both ligands involving identical Fab atoms, only about half are of similar types, i.e., polar-polar (including four hydrogen bonds, described below), nonpolar-nonpolar, or polarnonpolar. Nine residues (four in the light chain and five in the heavy chain) make substantial contact solely with the octapep- 
A
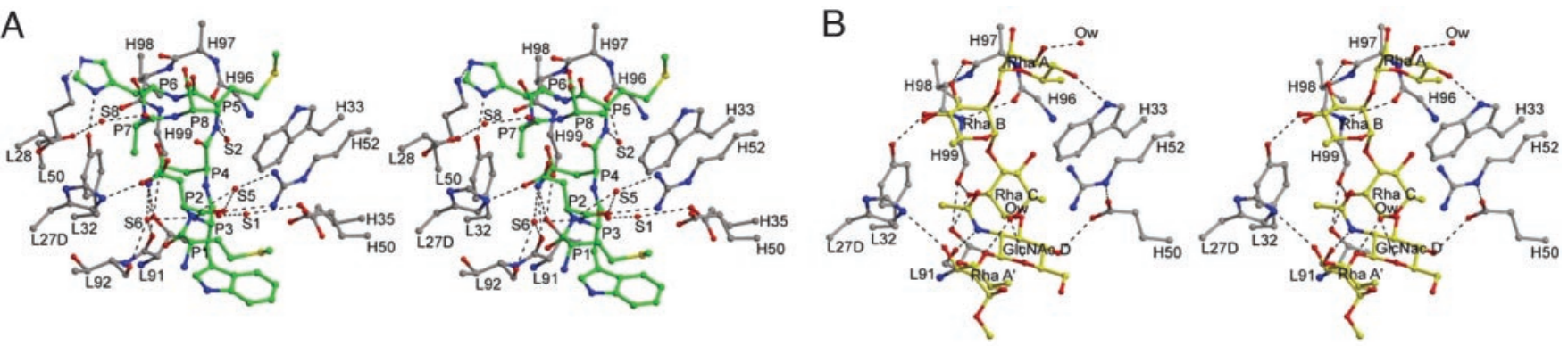

C

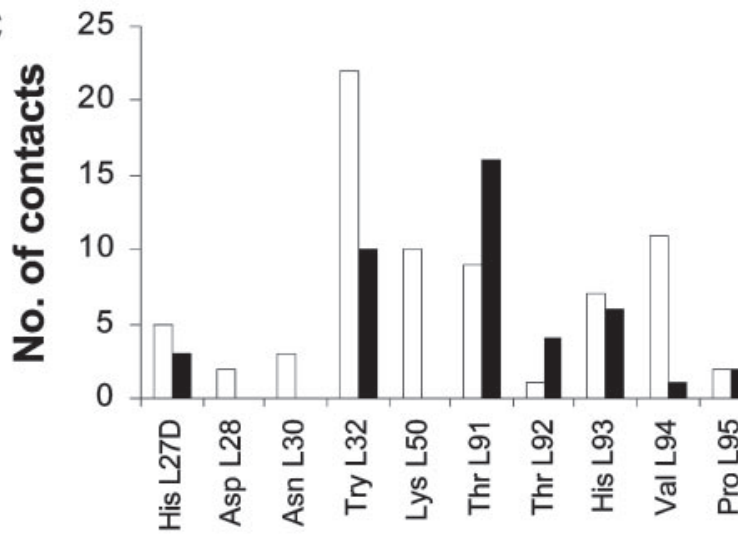

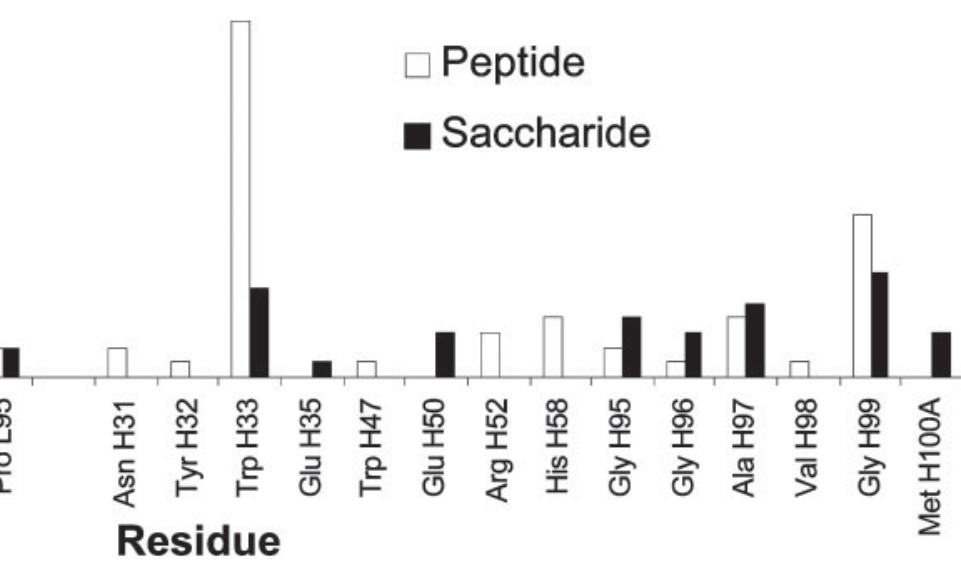

Fig. 3. Interactions between the Fab and octapeptide or pentasaccharide. (A) Stereoview of octapeptide binding, which includes eight direct and nine one-water-mediated hydrogen bonds. Note that 10 water molecules participate in bridging a total of 14 hydrogen bonds. Not shown are the following five two- or three-water-mediated hydrogen bonds: Met P1 O-S12-S3-Val L94 N, Met P1 O-S12-S11-His L93 ND1, Asp P2 OD1-S14-S8-Asp L28 OD1, Asp P2 $\mathrm{OD} 2-\mathrm{S} 13-\mathrm{S} 8-\mathrm{Arg} \mathrm{H} 52 \mathrm{NH}_{2}$, and Trp P3 O-S1-S9-S2-Gly H96 O. (B) Stereoview of pentasaccharide binding adapted from Vyas et al. (11). The two water molecules (Ow) bind solely to the sugar. (C) Comparison of the number of direct contacts of residues between the Fab and octapeptide or pentasaccharide (11). The numbers of direct hydrogen bonds and van der Waals contacts ( $\leq 4 \AA)$ associated with peptide binding are 6 and 126, respectively, and with saccharide binding are 8 and 74 , respectively.

tide as compared with two residues with the pentasaccharide (Fig. 3C). Finally, of the five residues that make close to 10 or more contacts with either ligand, four (Tyr L32, Val L94, Trp H33, and Gly H99) form significantly more contacts with the octapeptide as contrasted to only one (Thr L91) with the pentasaccharide (Fig. 3C).

Second, of the six and eight direct hydrogen bonds to the peptide and saccharide (Fig. $3 A$ and $B$ ), only four are made with identical Fab groups. Fab residues His L27D NE2, Thr L91 OG1,
Thr L91 O, and Tyr L32 OH form hydrogen bonds with Rha A' 3-OH, Rha C 2-OH, GlcNAc D 2-NH, and Rha B 4-OH, respectively, and Asp P2 OD1, Asn P4 ND2, Asn P4 ND2, and His P6 ND1, respectively.

Third, 14 water molecules interact with the peptide (Figs. $2 B$ and $3 A$ ), whereas only two water molecules were detected in pentasaccharide binding (Fig. 3B) (11). Of the 14 water molecules, only four water molecules (S1, S2, S6, and S12) are located within $2 \AA$ of the positions of polar atoms of the superposed
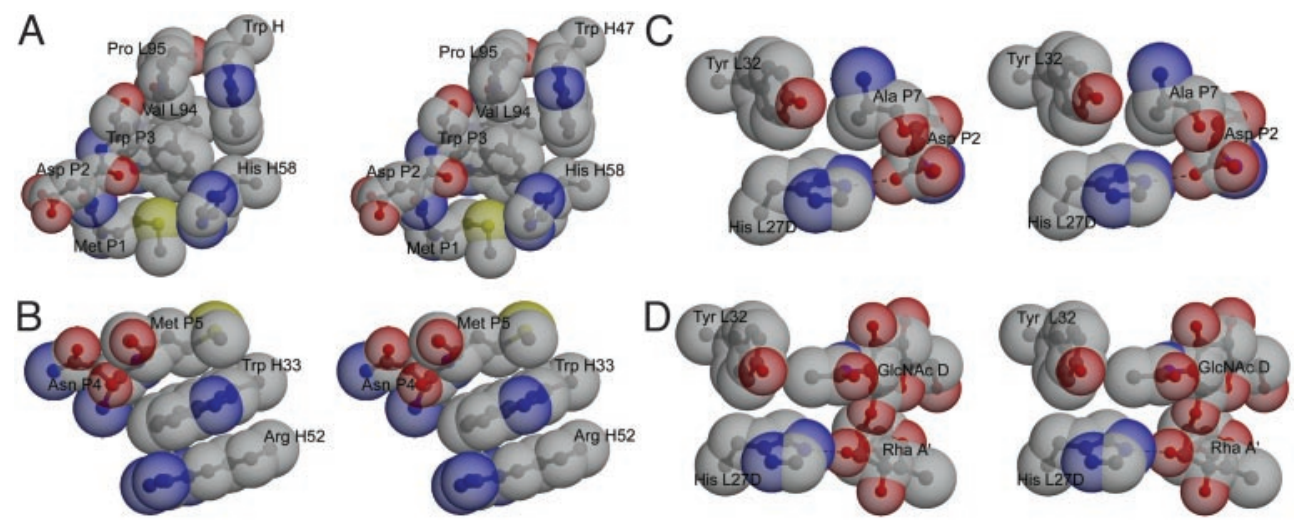

Fig. 4. Stereoviews (in transparent space-filling models) of the nonpolar interactions of the Fab with the peptide and pentasaccharide. ( $A$ ) Trp P3 in a nonpolar cavity (see also Fig. $1 B$ ). (B) Interactions of Met P5 with Trp H33. ( $C$ and $D$ ) Interactions of the octapeptide and pentasaccharide, respectively, with Tyr L32 and His L27D. In C, the methyl side chain of Ala P7 is close to Tyr L32, whereas side chains of Asp P2 and His L27D make electrostatic interaction. In D, the GIcNAC D acetamido group's methyl group forms nonpolar interaction with Tyr L32, whereas the Rha A' C3-OH hydrogen bonds with the His L27D side chain. 
pentasaccharide (Rha C 5-O, Rha C 4-OH, GlcNAc D 2-NH, and Rha $A^{\prime} 1-O$, respectively) (Fig. 2B). Because three of the saccharide's polar groups ( $\mathrm{Rha} \mathrm{C} 5-\mathrm{O}$ and $4-\mathrm{OH}$ and $\mathrm{Rha} \mathrm{A}^{\prime} \mathrm{O} 1$ ) do not participate in hydrogen-bonding interactions with the Fab (11), the role of the approximately equivalent water molecules (S1, S2, and S12, respectively) is likely unique to the binding of the octapeptide.

Fourth, the deep pocket in the combining site groove is filled by $\mathrm{Rha} \mathrm{C}$ of the pentasaccharide (Figs. $1 C$ and $2 B$ ). In contrast, the pocket, which is barely occupied by the peptide, is filled by a string of three hydrogen-bonded water molecules (S1, S2, and S9) (Figs. $1 B$ and $2 B$ ).

Carbohydrate-Peptide Mimicry. It has been suggested that peptidecarbohydrate mimicry originates in mimicry of specific chemical groups of the carbohydrate by chemical groups of the peptide (structural mimicry) (27-29). Some structural mimicry has been observed in the complexes of several mimetic proteins, e.g., in the complex of a camel heavy-chain antibody against lysozyme, where parts of some residues of the antibody mimic parts of the sugar substrate of lysozyme (30), and in the complex of porcine pancreatic $\alpha$-amylase with the proteinaceous inhibitor, where several specific hydrogen-bonding and hydrophobic interactions with the carbohydrate substrate are mimicked by the inhibitor (31). The concept of structural mimicry is also closely related to the immune network theory of Jerne and the concept of antiidiotypic antibodies (32). In principle, antiidiotypic antibodies structurally mimicking antigen will carry its "internal image," as supported by structural evidence with protein antigens $(33,34)$. In recent years, the question of mimicry with ligands of different molecular classes has been interpreted in terms of the nature of the involvement of the protein receptor $(12,33)$. Specifically, if two ligands are bound by similar groups on a protein, then this constitutes structural mimicry. If, however, they are bound by using different groups on the protein, then the nature of mimicry is functional. Our structural results, as described above and discussed further below, provide the first concrete evidence that the modes of binding of the pentasaccharide and octapeptide in the same antibody-combining site groove differ significantly, but few aspects of structural mimicry exist.

The selection of the octapeptide mimic of the O-antigen polysaccharide against which the SYA/J6 was raised by screening of phage-displayed libraries does not reflect a binding mode that faithfully replicates that of the pentasaccharide antigen. Rather, the selection is chiefly the consequence of two major factors: $(i)$ the bound octapeptide complements the shape of the antigen-binding site groove much better than does the pentasaccharide, and (ii) it uses to a much greater extent two primary types of interactions (van der Waals/nonpolar interactions and direct or water-mediated hydrogen bonds) with residues in the groove.

The Sc value, a measure of shape complementarity between interfacing surfaces (35), for the complex with the peptide $(0.77)$ is better than that with the pentasaccharide antigen (0.67). The inclusion of the 14 water molecules associated with peptide binding further enhances the shape complementarity $(\mathrm{Sc}=$ 0.82 ). This enhancement is essentially attributed entirely to the presence of S1, S2, and S9 at the interface between the peptide and the deepest part of the combining-site groove (Figs. $2 B$ and $3 A$ ), because the $\mathrm{Sc}$ value is unchanged by leaving in only the three water molecules. The lower Sc value of the interface with the oligosaccharide antigen is comparable to those of interfaces between Fab and other antigens (including proteins), which range from 0.64 to 0.68 (35). In contrast, the more favorable shape complementarity of the SYA/J6 Fab-peptide interface is also observed in protein oligomeric and protein-protein inhibitor interfaces (35). The appreciably greater shape complementarity of the complex with the octapeptide than with the pen- tasaccharide is consistent with the observation of a significantly larger number of intermolecular contacts in the complex with the peptide than with the pentasaccharide (described above).

As similarly observed in the complex with the pentasaccharide (11), hydrophobic interactions are also heavily involved in the binding of the octapeptide. However, only a few of the extensive hydrophobic interactions associated with pentasaccharide binding is mimicked to some extent by the peptide. The acetamido methyl group of GlcNAc D is buried entirely in a small hydrophobic cavity (Fig. 4D), whereas the methyl side chain of Ala P7 binds only partially in this cavity (Fig. $4 C$ ). The 6-methyl group of Rha $\mathrm{C}$ of the pentasaccharide, which is located in the deep cavity at the bottom of the groove (Fig. $1 C$ ), makes hydrophobic interactions with Met H100A and Thr L91 (11). These interactions are not mimicked by the octapeptide because it does not penetrate the cavity. Instead, three water molecules (S2, S9, and S1) occupy the hole (Figs. $1 B$ and $2 B$ ). However, the lack of mimicry is overcome by other hydrophobic interactions unique to the octapeptide side chains (Figs. $1 B$ and $4 A$ and $B$ ), for example, Met P1 and Met P5, and the burial of Trp P3 in a shallow hydrophobic cavity.

Hydrogen bonds, which are highly directional, represent the second important set of interactions because they mainly confer the specificity in binding of the peptide and the carbohydrate antigen. However, as described above, direct hydrogen bonds constitute only a very small fraction of the contacts between the Fab and octapeptide or pentasaccharide (Fig. 3C). Furthermore, of the six and eight such hydrogen bonds with the octapeptide and pentasaccharide, respectively, only four hydrogen bonds may be considered as being similar (described above).

The hydrogen bonds mediated by numerous water molecules represent the most striking feature of the Fab-peptide complex. A paramount question is whether the water molecules mimic the hydroxyl groups of the oligosaccharide. Examination of the Fab-octapeptide complex structure provides no strong evidence for this claim. Rather, we suggest that the water molecules fulfil the following critical functions in the octapeptide binding. First, they fill voids between the peptide and Fab. This function is best exemplified by S1, S9, and S2, which form a string of hydrogen bonds and fill the cavity at the bottom of the groove (Figs. $1 B$ and $2 B)$. In contrast to the peptide binding, Rha $\mathrm{C}$ of the pentasaccharide fills the cavity (Figs. $1 C$ and $2 B$ ). The arrangement of the three water molecules, together with that of the peptide backbone (from the $\mathrm{C}=\mathrm{O}$ bond of Trp P3 to the NH of Met P5), is similar to the shape of Rha C (Fig. $2 B$ ). This is a clear example of shape mimicry involving water molecules and parts of the peptide for a sugar residue. Second, the water molecules bridge, through hydrogen bonds, the octapeptide and Fab. When these bridging interactions constitute as much as 14 hydrogen bonds involving nine water molecules (Fig. $3 A$ ), which are eight more than the direct hydrogen bonds between Fab and octapeptide (Fig. $3 B$ ), they can contribute to the specificity of complex formation. No bridging water is observed in the complex with the saccharide. Third, nine water molecules link as many as five hydrogen bonds between residues within the octapeptide (Fig. $2 B$ ), thus contributing to stability of the binding-competent conformation of the peptide.

Despite the better shape complementarity and greater number of contacts associated in the binding of the octapeptide, its association constant $\left(K_{\mathrm{A}}=2.5 \times 10^{5} \mathrm{M}^{-1}\right)$ determined by isothermal titration calorimetry is similar to that of the pentasaccharide epitope binding $\left(K_{\mathrm{A}}=5.7 \times 10^{5} \mathrm{M}^{-1}\right)(11)$. However, the binding of the pentasaccharide is accompanied by favorable enthalpy and entropy, with the latter exceeding the former $\left(\Delta H=-1.5 \mathrm{kcal} \cdot \mathrm{mol}^{-1}\right.$ and $\left.-T \Delta S=-5.9 \mathrm{kcal} \cdot \mathrm{mol}^{-1}\right)$, which is consistent with the major role of hydrophobic interactions (11). In contrast, the octapeptide binding is strongly enthalpy-driven, with considerable unfavorable entropy $(\Delta H=$ 
$-16.9 \mathrm{kcal} \cdot \mathrm{mol}^{-1}$ and $\left.-T \Delta S=+9.1 \mathrm{kcal} \cdot \mathrm{mol}^{-1}\right)$. Presumably, the more favorable and dominant enthalpic term is due to the larger number of peptide-Fab contacts, and the unfavorable entropy arises from a more ordered bound peptide relative to the unbound form (with imposition of one $\alpha$-helical turn) and the immobilization of many water molecules. NMR analysis indicates that the free peptide is a random coil (unpublished data).

Regarding vaccine development, we view this and other carbohydrate-mimetic peptides as lead candidates. The knowledge gained from the structural work will guide further development for increasing peptide affinity through modifications to enhance helicity, to exploit the deep hydrophobic pocket in the middle of the groove, and to minimize or eliminate the competing, deleterious effect of the association of several water molecules, especially with both exposed termini of the octapeptide. Because the octapeptide is a functional mimic of the cell-surface polysaccharide epitope of S. flexneri, it would be of interest to pose the question of whether a conjugated peptide will elicit an antipolysaccharide response. In addition, the absence of structural mimicry might not preclude use of the peptide in a vaccine protocol. Thus, although it is possible that, by itself, the peptide conjugate will not elicit an antipolysaccharide response, it could still be used in a prime/boost strategy to enhance the immune response to an already-present carbohydrate epitope, as demonstrated recently for the case of a peptide mimic of the capsular polysaccharide of Cryptococcus neoformans (36).

1. Jennings, H. J. (1988) Adv. Exp. Med. Biol. 228, 495-550.

2. Wolchok, J. D. \& Livingston, P. O. (2001) Lancet Oncol. 2, 205-211.

3. Lindberg, A. A. (1999) Vaccine 17, Suppl. 2, S28-S36.

4. Alexander, J., del Guercio, M. F., Maewal, A., Qiao, L., Fikes, J., Chesnut, R. W., Paulson, J., Bundle, D. R., DeFrees, S. \& Sette, A. (2000) J. Immunol. 164, 1625-1633.

5. Johnson, M. A. \& Pinto, B. M. (2002) Aust. J. Chem. 55, 13-25.

6. Zwick, M. B., Shen, J. \& Scott, J. K. (1998) Curr. Opin. Biotechnol. 9, 427-436.

7. Cunto-Amesty, G., Luo, P., Monzavi-Karbassi, B. \& Kieber-Emmons, T. (2001) Int. Rev. Immunol. 20, 157-180.

8. Monzavi-Karbassi, B., Cunto-Amesty, G., Luo, P. \& Kieber-Emmons, T. (2002) Trends Biotechnol. 20, 207-214.

9. Pincus, S. H., Smith, M. J., Jennings, H. J., Burritt, J. B. \& Glee, P. M. (1998) J. Immunol. 160, 293-298.

10. Bundle, D. R. (1989) Pure Appl. Chem. 61, 1171-1180.

11. Vyas, N. K., Vyas, M. N., Chervenak, M. C., Johnson, M. A., Pinto, B. M., Bundle, D. R. \& Quiocho, F. A. (2002) Biochemistry 41, 13575-13586.

12. Harris, S. L., Craig, L., Mehroke, J. S., Rashed, M., Zwick, M. B., Kenar, K., Toone, E. J., Greenspan, N., Auzanneau, F. I., Marino-Albernas, J. R., et al. (1997) Proc. Natl. Acad. Sci. USA 94, 2454-2459.

13. Vyas, M. N., Vyas, N. K., Meikle, P. J., Sinnott, B., Pinto, B. M., Bundle, D. R. \& Quiocho, F. A. (1993) J. Mol. Biol. 231, 133-136.

14. Otwinowski, Z. \& Minor, W. (1997) Methods Enzymol. 276, 307-326.

15. Brunger, A. T., Adams, P. D., Clore, G. M., DeLano, W. L., Gros, P., Grosse-Kunstleve, R. W., Jiang, J. S., Kuszewski, J., Nilges, M., Pannu, N. S., et al. (1998) Acta Crystallogr. D 54, 905-921.

16. Lawskowski, R. A., McArthur, M. W., Moss, D. S. \& Thorton, J. M. (1993) J. Appl. Crystallogr. 26, 282-291.

17. Ferrin, T., Huang, C., Jarvis, L. \& Langridge, R. (1988) J. Mol. Graphics 6, 13-27.

18. Nicholls, A., Sharp, K. A. \& Honig, B. (1991) Proteins Struct. Funct. Genet. 11, 281-296.

\section{Conclusions}

Our x-ray structure analysis of the binding of the octapeptide mimic and the natural $\mathrm{O}$-antigen pentasaccharide antigen to the combining site of the SYA/J6 Fab shows that the nature of the mimicry is mainly functional, i.e., molecular recognition of both ligands differs significantly. The octapeptide makes significantly greater shape complementarity and number of contacts with the combining site than the pentasaccharide. Of these contacts (126 and 74 with the peptide and saccharide, respectively), which are mostly van der Waals interactions, an overwhelming number are considered components of functional mimicry. Only 18 contacts, including four hydrogen bonds, represent structural mimicry. A striking feature unique to the Fab-octapeptide complex is the involvement of 12 water molecules in mediating hydrogen bonds between residues within the peptide or of the peptide and Fab. This type of hydrogen bond not only confers specificity and affinity to the complex but also stability to the conformation of the peptide.

This work is dedicated to the memory of Professor R. U. Lemieux. We thank X. Duan, W. E. Meador, and J. Sadowska for technical assistance, C. Ogata for assistance in data collection at the Howard Hughes Medical Institute Brookhaven National Laboratory beamline, and M. A. Johnson for helpful discussion. This work was supported by grants from the National Institutes of Health (to N.K.V. and F.A.Q.), the Welch Foundation (to F.A.Q.), and the Natural Sciences and Engineering Research Council of Canada (to D.R.B. and B.M.P.).

19. Kraulis, P. (1991) J. Appl. Crystallogr. 24, 946-950.

20. Harris, S. L. (1997) Ph.D. dissertation (Simon Fraser University, Buraby, BC, Canada).

21. Richardson, J. S. \& Richardson, D. C. (1989) in Prediction of Protein Structures and the Principles of Protein Conformation, ed. Fasman, G. D. (Plenum, New York), pp. 2-98.

22. Dougherty, D. A. (1996) Science 271, 163-168.

23. Cygler, M., Rose, D. R. \& Bundle, D. R. (1991) Science 253, 442-445.

24. Jeffrey, P. D., Bajorath, J., Chang, C. Y., Yelton, D., Hellstrom, I., Hellstrom, K. E. \& Sheriff, S. (1995) Nat. Struct. Biol. 2, 466-471.

25. Herron, J. N., He, X. M., Mason, M. L., Voss, E. W., Jr., \& Edmundson, A. B. (1989) Proteins Struct. Funct. Genet. 5, 271-280.

26. Wilson, I. A. \& Stanfield, R. L. (1994) Curr. Opin. Struct. Biol. 4, 857-867.

27. Agadjanyan, M., Luo, P., Westerink, M. A., Carey, L. A., Hutchins, W., Steplewski, Z., Weiner, D. B. \& Kieber-Emmons, T. (1997) Nat. Biotechnol. 15, 547-551.

28. Kieber-Emmons, T., Luo, P., Qiu, J., Agadjanyan, M., Carey, L., Hutchins, W. Westerink, M. A. \& Steplewski, Z. (1997) Hybridoma 16, 3-10.

29. Lou, Q. \& Pastan, I. (1999) J. Pept. Res. 53, 252-260.

30. Transue, T. R., De Genst, E., Ghahroudi, M. A., Wyns, L. \& Muyldermans, S. (1998) Proteins 32, 515-522.

31. Bompard-Gilles, C., Rousseau, P., Rouge, P. \& Payan, F. (1996) Structure (London) 4, 1441-1452.

32. Jerne, N. K. (1974) Ann. Immunol. (Paris) 125C, 373-389.

33. Fields, B. A., Goldbaum, F. A., Ysern, X., Poljak, R. J. \& Mariuzza, R. A. (1995) Nature 374, 739-742.

34. Mariuzza, R. A. \& Poljak, R. J. (1993) Curr. Opin. Immunol. 5, 50-55.

35. Lawrence, M. C. \& Colman, P. M. (1993) J. Mol. Biol. 234, 946-950.

36. Beenhouwer, D. O., May, R. J., Valadon, P. \& Scharff, M. D. (2002) J. Immunol. 169, 6992-6999. 